\title{
UMA COMPARAÇÃO ENTRE AS ANÁLISES MACROECONÔMICAS E OS PARÂMETROS FISCAIS BRASILEIRO ENTRE OS ANOS DE 2010 A 2019
}

\section{A COMPARATION BETWEEN MACRONOMICS ANALYSIS AND BRAZILIAN FISCALS PARAMETERS IN THE YEARS 2010 TO 2019.}

\section{Charleston Sperandio de Souza, Mestre e Professor de Administração e Economia pela Alfa Unipac, Aimorés, Brasil. E-mail: charleston.sperandio@yahoo.com.br}

Antônio Alexandre Dias Junior, Administrador, egresso 2019 da Alfa Unipac, Aimorés, Brasil.

E-mail: diasjuniorr@hotmaill.com

Jonathan Laranjeira Saraiva da Silva, Acadêmico do $8^{\circ}$ período de Administração da Alfa Unipac, Aimorés, Brasil.

E-mail: jonathan.saraiva@gmail.com

Recebido: 10/11/2020 - Aceito: 27/11/2020

\section{Resumo}

O presente estudo teve como objetivo analisar o cenário macroeconômico do Brasil no período entre os anos de 2010 a 2019, bem como apresentar as motivações que geraram uma crise econômica e financeira no País. A economia brasileira, no período entre 2010 e 2014, teve crescimento médio de 2,8\% ao ano. Esse período foi marcado, pelas consequências da crise internacional. A partir de 2015 e 2016, a economia brasileira passou por um período de recessão. Os recuos foram de $-3,5 \%$, para 2015 e 2016, em termos anuais. No ano de 2017, a economia apontou para uma razoável recuperação econômica, depois de uma recessão caracterizada no biênio 2015/2016. No ano de 2018, com o bom comportamento do consumo das famílias, o PIB cresceu $1 \%$ e a economia desenvolveu-se rapidamente. Já em 2019 o PIB teve um acréscimo de $1,1 \%$ seguindo a expansão de 2017 e 2018 , e a inflação foi de $4,31 \%$ e os juros a longo prazo tiveram uma redução nesse período; e a taxa de desemprego em 2019 caiu $0,4 \%$ em relação a 2018 , fechando o ano em $11,9 \%$. A metodologia teve uma análise das medidas de tendência central com abordagem nos parâmetros econômicos e fiscais com análise qualitativa, com um corte transversal e com ênfase nos dados que foram analisados em um intervalo de tempo; e foi descritiva, pois destinou-se a descrever as características de determinada situação. Os resultados apontaram que o país passou por crise econômica de 2013 a 2016 e que a partir de 2017 até 2019 houve uma recuperação gradual. Conclui-se que a economia brasileira passou por anos de crises e instabilidades, o que resultou em Inflação alta, baixa da moeda, taxas de desemprego elevadas, e de acordo com os resultados, a tendência para os anos subsequentes é uma recuperação em todos os aspectos.

Palavras-chave: Economia brasileira; parâmetros fiscais; inflação. 


\section{Abstract}

This study aimed to analyze the macroeconomic scenario of Brazil in the period between 2010 and 2019, as well as to present the motivations that generated an economic and financial crisis in the country. The Brazilian economy, in the period between 2010 and 2014, had an average growth of $2.8 \%$ per year. This period was marked by the consequences of the international crisis. From 2015 to 2016, the Brazilian economy went through a period of recession. The decreases were $-3.5 \%$ for 2015 and 2016 in annual terms. In 2017, the economy pointed to a reasonable economic recovery, after a recession characterized in the biennium 2015/2016. In 2018 , with the good behavior of household consumption, PIB grew by $1 \%$ and the economy developed rapidly. In 2019 , PIB increased by $1.1 \%$ following the expansion of 2017 and 2018 , and inflation was $4.31 \%$ and long-term interest rates fell during this period; and the unemployment rate in 2019 fell $0.4 \%$ compared to 2018 , closing the year at $11.9 \%$. The methodology had an analysis of the measures of central tendency with approach in economic and fiscal parameters with qualitative analysis, with a crosssectional and with emphasis on the data that were analyzed in a time interval; and was descriptive, as it was intended to describe the characteristics of a given situation. The results showed that the country went through an economic crisis from 2013 to 2016 and that from 2017 to 2019 there was a gradual recovery. It is concluded that the brazilian economy has experienced years of crises and instabilities, which resulted in high inflation, low currency, high unemployment rates, and according to the results, the trend for subsequent years is a recovery in all aspects.

Key Word: Brazilian economic; tax parameters; and inflantion.

\section{Introdução}

$\mathrm{Na}$ atualidade, a relação entre a economia, as organizações públicas, privadas e a sociedade, são características cada vez mais associadas aos estudos dos economistas objetivando o desenvolvimento econômico.

O desenvolvimento econômico é conceituado como a evolução do bem-estar da população de modo geral, apontado pela ascensão dos identificadores quantitativos da economia brasileira, como o Produto Interno Bruto (PIB) por exemplo, e é ainda anunciado para uma melhoria dos indicadores qualitativos a respeito da qualidade de vida da população brasileira.

De acordo com a economia, existe uma diferença entre o crescimento econômico e o desenvolvimento econômico. O crescimento econômico é mais fácil de ser alcançado. Ele pode ser acentuado como a melhora dos indicadores quantitativos 
da economia, como por exemplo, o aumento da renda, a redução do desemprego ou o aumento do consumo. Por outro lado, o desenvolvimento econômico aborda um conceito mais abarcante.

Pode-se inferir deste modo, que o desenvolvimento envolve o crescimento econômico, ou seja, não existe um desenvolvimento sem o crescimento, porém, nem sempre que há crescimento pode ser considerado que existiu o desenvolvimento.

De acordo com esse contexto, o Brasil após um período de desenvolvimento econômico entre os anos de 2004 a 2013, teve uma taxa de crescimento anual médio de 4,0\% ao ano, seguido por uma aceleração no progresso da distribuição de emprego e renda.

No entanto, a partir de 2014, a economia brasileira entrou em uma forte e prolongada recessão entre os anos de 2015 e 2016, com uma taxa de desenvolvimento do PIB, tendo uma média negativa em -3,7\%, (menos três vírgula sete por cento) provocando uma grave crise econômica em vários indicadores sociais, prejudicando a todos os brasileiros, principalmente as classes menos favorecidas, ou seja, a classe pobre do país (PAULA; PIRES, 2017).

Dessa forma, o ano de 2017 apontou na economia brasileira como o princípio de uma recuperação econômica, depois da agressiva recessão que caracterizou o biênio 2015/2016. Com o bom comportamento do consumo das famílias, o PIB cresceu positivamente 1\%, admitindo boas expectativas e recuperação para 2018 quando a economia finalmente voltou a crescer (TINOCO; GIAMBIAGI, 2018), e ainda no ano de 2018, segundo ano pós-recessão, a economia desenvolveu-se progressivamente mais do que o ano anterior, mostrando que a recuperação foi lenta e gradual (BALASSIANO, 2018) e o autor informa ainda que em 2019 o PIB brasileiro teve um acréscimo de 1,1\% seguindo a expansão de 2017 e 2018, somando em valores correntes $R \$ 7,3$ trilhões no período. Comparativamente com o quarto trimestre de 2018 o PIB cresceu 1,7\%.

Assim, a proposta deste trabalho tem como objetivo principal analisar o cenário macroeconômico do Brasil no período entre os anos de 2010 até 2019, e comparar os seguintes indicadores econômicos: Taxas de Juros (SELIC - Sistema Especial de Liquidação e de Custódia para títulos federais), IPCA (Índice Nacional de Preço ao Consumidor) e a Taxa de desemprego entre si, bem como apresentar as motivações econômicas que geraram a crise financeira. 
Justifica-se a presente pesquisa, por entender que a relevância se consiste em contribuir com o tema em questão e que irá subsidiar o entendimento acerca do mercado, no cenário macroeconômico e seus impactos gerados à sociedade.

\subsection{A Economia Brasileira no Período de 2010 A 2019}

A economia brasileira no período compreendido entre 2010 e 2014, teve um crescimento médio de 2,8\% ao ano. Esse período foi marcado, pelas consequências da crise internacional e pela ligeira recuperação do Brasil. Posteriormente, o período se retraiu pela desaceleração do crescimento econômico, que foi motivado tanto pela falta de estabilidade no ambiente externo, quanto às medidas intervencionistas adotadas pelo governo a fim de evitar uma desaceleração ainda maior na economia (TINOCO; GIAMBIAGI, 2018).

Dessa forma, acumularam-se os desequilíbrios na economia. A inflação se manteve obstinadamente perto do limite superior. O déficit em conta corrente chegou a 4,2\% do PIB (TINOCO; GIAMBIAGI, 2018), porém de acordo com Barbosa Filho (2014), mesmo com o aumento do PIB, houve uma brusca desaceleração de seu crescimento nesse período.

A partir de 2015 e 2016, a economia brasileira passou por um período de recessão. Os recuos foram de -3,5\% em 2015 e 2016 em termos anuais. Já em termos trimestrais, tiveram queda em todos os trimestres, com evidência negativa para 0 segundo trimestre de 2015, quando a economia declinou nas casas dos 1,9\% (BARBOSA FILHO, 2014).

A intensa retração econômica do período dessa recessão, ocasionou desgaste de todo o cenário macroeconômico, prejudicando as empresas e famílias, diminuindo drasticamente o consumo de bens e serviços, e com conflito em uma série de indicadores. No mercado de trabalho o agravamento aumentou, e esse cenário cooperou para reforçar a queda do consumo das famílias (CEMEC, 2016).

Os dados do IBGE - Instituto Brasileiro de Geografia e Estatística, avaliado pelo Sistema de Contas Nacionais Trimestrais (IBGE-SCNT, 2015) apontaram que no setor externo, as exportações de Bens e Serviços tiveram aumento de $+3,4 \%$ no segundo trimestre de 2015, enquanto que as importações de Bens e Serviços recuaram -8,8\% em relação ao primeiro trimestre. 
No ano de 2017, finalmente a economia do Brasil interrompeu o ciclo de dois anos de recessão e da queda do PIB. O crescimento foi de $+1 \%$, com elevação em todos os trimestres no decorrer do ano (IBGE-SCNT, 2017).

Múltiplos fatores colaboraram para o crescimento da economia, principalmente a política monetária cada vez mais expansionista, como a safra agrícola positiva e também uma situação internacional favorável (GIAMBIAGI; ALMEIDA, 2017).

Em termos de oferta, a grande ênfase veio da agropecuária, com alta de 13\%, favorecendo-se da safra positiva (a mais alta da história). Em termos de demanda, a ênfase foi o consumo das famílias, que exibiu alta em todos os trimestres do ano e fechou 2017, com elevação de +1\% (IBGE-SCNT, 2017).

Porém, em janeiro de 2018, o relatório Focus ${ }^{1}$, produzido pelo Banco Central, apontou perspectiva de desenvolvimento da atividade econômica brasileira de cerca de $+3 \%$. Contudo, devido ao cenário inconstante, tendo, por exemplo, a greve dos caminhoneiros, o tabelamento de fretes e a incerteza eleitoral, a esperança de crescimento para o ano de 2018 desmoronou para -1,3\%, de acordo com o mesmo relatório da primeira semana de dezembro (MARTINS et al, 2018).

A respeito da greve dos caminhoneiros:

[...] teve efeitos diretos sobre o PIB e indiretos, por meio da piora das expectativas. Adicionalmente, o agravamento da economia internacional e a proximidade das eleições no Brasil contribuíram para formar um quadro de elevado nível de incertezas, que prejudicou a retomada da economia no primeiro semestre do ano (CARVALHO, 2018, p. 2)

Enquanto isso, a inflação voltou a ganhar força. O IPCA elevou para $+1,26 \%$, sendo a maior taxa para o mês de junho de 2018, desde 1995 (TREVIZAN, 2018). Ainda de acordo com o autor, em agosto de 2018, a economia brasileira teve desenvolvimento de $+0,47 \%$ em relação a junho, segundo informações divulgadas pelo Banco Central do Brasil.

O cálculo do banco é realizado por meio do indicador IBC-BR (Índice de Atividade Econômica do Banco Central). O resultado ficou acima da expectativa em

\footnotetext{
${ }^{1} \mathrm{O}$ Relatório Focus resume as estatísticas calculadas considerando as expectativas de mercado coletadas até a sexta-feira anterior à sua divulgação. Ele é divulgado toda segunda-feira. O Relatório traz a evolução gráfica e o comportamento semanal das previsões para índices de preços, atividade econômica, câmbio, taxa Selic, entre outros indicadores.
} 
pesquisa da Reuters ${ }^{2}$ de alta de $+0,25 \%$. Na comparação do mesmo mês do ano anterior a 2018, o avanço foi de $+2,5 \%$, segundo matéria da Folha de São Paulo (2018).

Ainda com a taxa de desemprego em alta e a confiança dos empresários e consumidores em baixa, a economia brasileira mostrou um ritmo de recuperação mais lento que inicialmente esperado para 2018. A indústria, comércio e serviços exibiram desempenhos distintos em agosto de 2018. A produção industrial caiu $-0,3 \%$ em agosto, sendo a segunda queda mensal seguida. Já as vendas do comércio cresceram $+1,3 \%$ em agosto de 2018, após 3 quedas seguidas (SILVEIRA; ALVARENGA, 2018).

O relatório da Agência IBGE (2020) revelou que o IPCA terminou o ano de 2018 com 3,75\% e o mesmo teve um aumento no ano de 2019 terminando o mês de dezembro com 4,31\%, onde o seu percentual no mês de dezembro de 2019 foi de 1,15\% e em novembro de 2019 foi apontado com um percentual de 0,51\%. De acordo com esse percentual, pode-se notar que foi um percentual superior desde o mês de dezembro no exercício de 2002, onde o IPCA ficou em 2,10\%.

Dessa forma, o relatório Focus (2019) revelou que a inflação de 2019 foi de $4,31 \%$ e os juros a longos prazos tiveram uma redução forte nesse período, o que não ocorria nos últimos anos, mostrando uma redução da percepção dos riscos do mercado doméstico e uma segurança na condução da política monetária. A inflação consolidada ficou próximo da meta e permitiu a redução das taxas de juros, como a SELIC que caiu 13,75\% a.a. (ao ano) de 2016 fechando em 2019 em 5,79\% a.a.

Por fim, a Agência Brasil (2019) relatou que a taxa de desemprego em 2019 caiu $0,4 \%$ em relação a 2018 fechando o ano em 11,9\%, girando com uma média de menos de 215 mil pessoas desempregadas em comparação ao ano de 2018. O acréscimo de pessoas trabalhando por conta própria foi de 4,1\% atingindo 24,2 milhões em comparação a 2018, enquanto o número de pessoas sem carteira assinada subiu 4\% significando mais 446 mil pessoas em 2019 em comparação a 2018.

\footnotetext{
2 É uma agência de notícias britânica, sendo a maior agência internacional de notícias do mundo, com sede em Londres.
} 


\section{Metodologia}

A presente pesquisa consistiu em uma análise do tipo de correlação das demonstrações macroeconômicas e dos parâmetros fiscais brasileiro entre os anos de 2010 a 2019, na qual se averiguou a relação entre as variáveis Taxas de Juros SELIC, IPCA e a Taxa de desemprego, que acontecem espontaneamente, sem a interferência das variáveis independentes, como por exemplo acontece em estudos de pesquisas experimentais de acordo com os autores Selltiz, Wrightsman \& Cook, (1976) e Contandriopoulos, et al, (1994).

Assim, o estudo teve uma abordagem nos parâmetros econômicos e fiscais com análise qualitativa e tratou-se, além disso, de uma pesquisa de corte transversal, com ênfase nos dados que foram analisados em um intervalo de tempo (CAMPBELL \& STANLEY, 1979). O tipo de pesquisa foi descritiva, pois destinou-se a apresentar as características de determinada circunstância. Em oposição ao que ocorre nos estudos exploratórios, a preparação dos questionamentos das pesquisas implica em profunda informação do problema a ser analisado.

O desenho das figuras 1, 2 e 3 dos dados, teve por finalidade representar os dados em percentuais adquiridos, consentindo aproximar-se das conclusões sobre 0 desenvolvimento ou sobre como se correlacionam as variáveis.

As colunas alusivas aos números nas tabelas 1, 2 e 3, contêm simultaneamente as frequências calculadas em porcentuais e as medidas de tendência central que visaram determinar o núcleo da distribuição dos dados notados.

A análise do histórico dos parâmetros fiscais referentes à economia brasileira possibilitará proporcionar um melhor entendimento dos resultados apresentados nas Taxas de Juros SELIC, IPCA e a Taxa de desemprego e assim, realizar as análises estatísticas das medidas de tendência central.

Na figura 1 a taxa de juros é operada com o objetivo de controlar a demanda agregada (SERRANO, 2010). Quando a taxa de juros sobe, veja nos anos de 2014, 2015 e 2016 (figura 1), os custos relacionados ao capital de giro aumentam, reduzindo as receitas das empresas. 
Figura 1. Parâmetros Fiscais da Taxa de Juros (SELIC) - \% ao ano.

TAXAS DE JUROS SELIC - \%

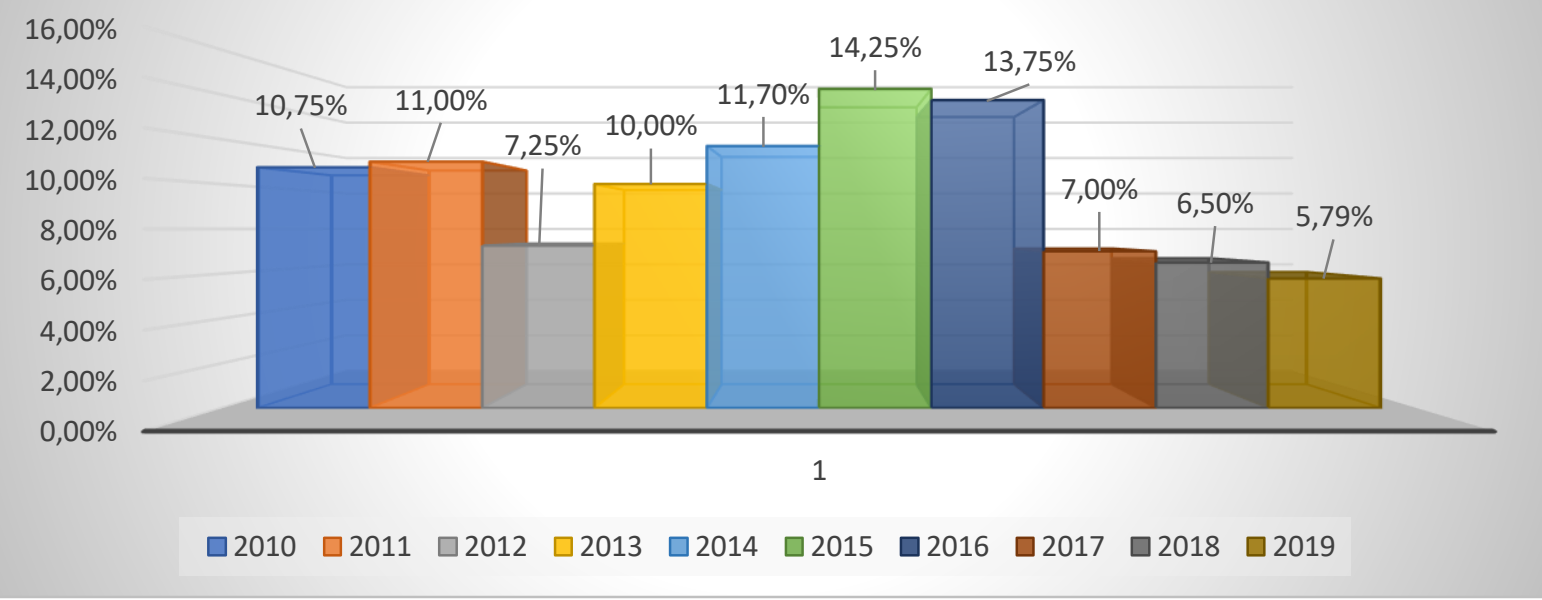

Fonte: IBGE (2019) - Realizada pelos autores.

Ao analisar a figura 1, observou-se que a taxa de juros caiu a partir de 2017 em relação dos anos anteriores analisados desde 2010.

Quando os juros sobem, o custo de oportunidade do capital também sobe. Com isso, os recursos são transferidos da produção para os títulos públicos, causando recessão (KANCZUK, 2002) e ao contrário quando os juros caem, as oportunidades de consumo aumentam, o que se demonstrou a partir de 2017.

$\mathrm{Na}$ figura 2, apresenta-se o IPCA que é o Índice Nacional de Preços ao Consumidor produzido pelo SNIPC (Sistema Nacional de Índices de Preços ao Consumidor), e o seu objetivo é medir para fins práticos, a inflação de produtos e serviços referentes ao consumo.

Figura 2 - Parâmetros Fiscais do IPCA em \% ao ano

IPCA POR ANO \%

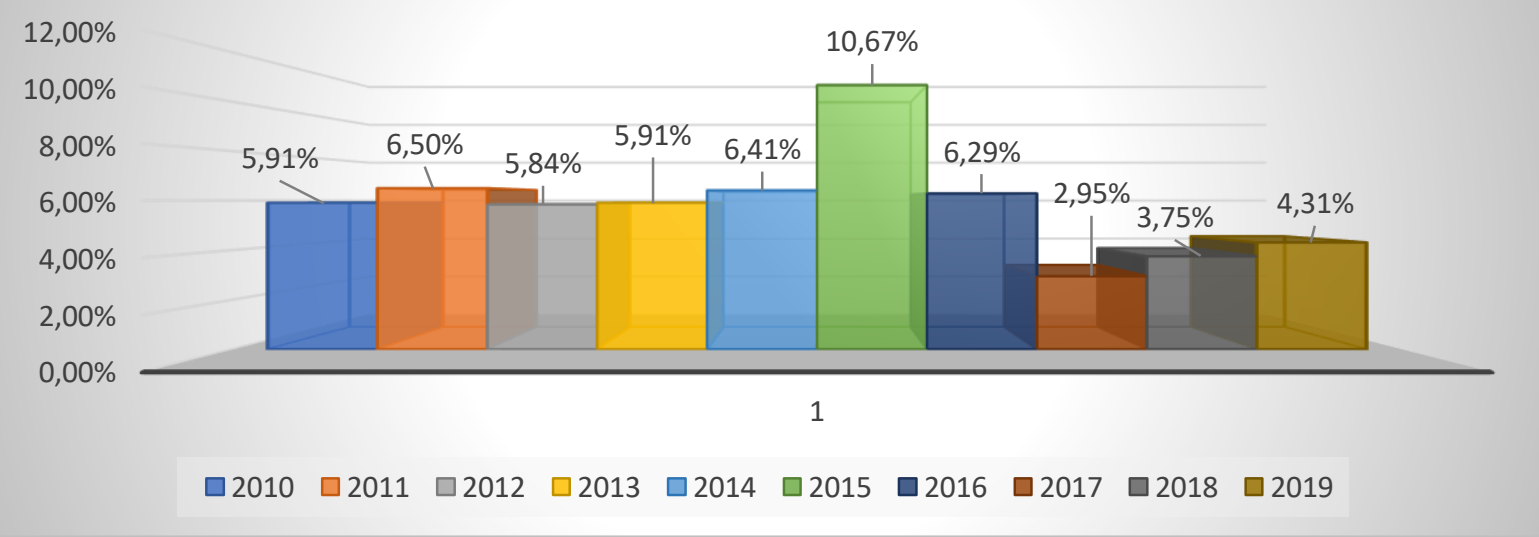


Fonte: IBGE (2019) - Realizada pelos autores.

Na figura 2, o IPCA demonstrou seu comportamento em decorrência da taxa de juros apresentada na figura 1, nos anos de 2014, 2015 e 2016, e pode-se perceber que o IPCA oscilou positivamente nos anos estimados, e consequentemente, teve uma pequena elevação dos produtos e serviços nos anos de 2017, 2018 e 2019.

$\mathrm{Na}$ figura 3 demonstra-se a taxa de desemprego, que menciona à desocupação ou desemprego no Brasil. Ela é verificada pelo IBGE e seus valores apresentados foram determinados a partir de estudos feitos a cada ano em relação à população economicamente ativa.

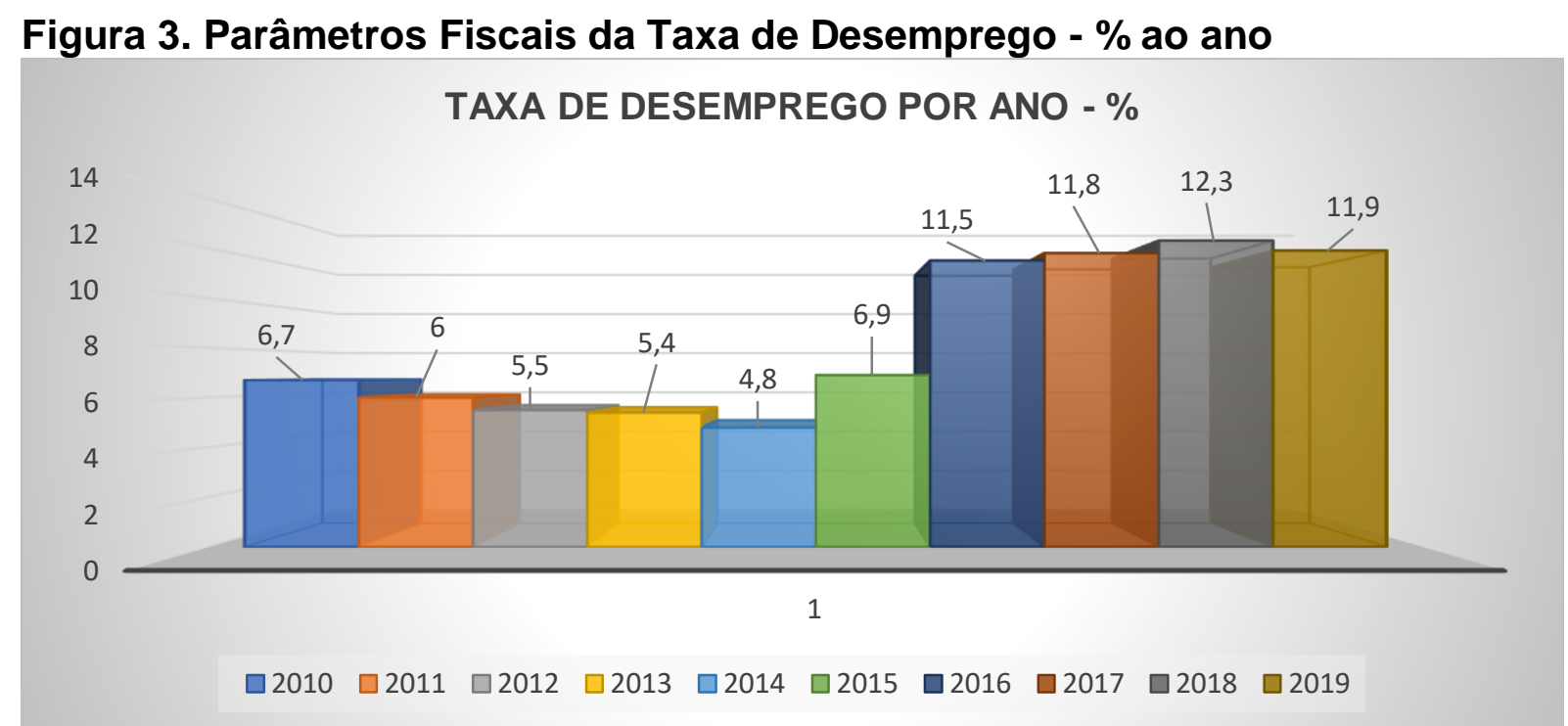

Fonte: IBGE (2019) - Realizada pelos autores.

$\mathrm{Na}$ análise da figura 3, a taxa de desemprego aumentou a partir de 2015 discretamente em relação aos anos anteriores, e disparou a partir dos anos de 2016, 2017 e 2018, representando no ano de 2018, aproximadamente mais de 13 milhões de desempregados no país, fechando em 2019 com um percentual inferior em relação ao ano anterior e ficando com a média anual de desocupados de aproximadamente 12,8 milhões de brasileiros (IBGE, 2019).

\section{Resultados e Discussão}


Nas tabelas 1, 2 e 3 a seguir, representam os resultados da medida de tendência central, que foram analisados dentro das medidas de dispersão, que são um conjunto de informações em relação à média aritmética.

Nesse cenário a intenção foi de apresentar a regularidade de acordo com os parâmetros fiscais apresentados anteriormente nas figuras 1, 2 e 3 .

Tabela 1. Medidas de Tendência Central das Taxas de Juros SELIC

\begin{tabular}{c|c|c|c|c|c|c|c|c}
\hline Ano & $\begin{array}{c}\text { Valores } \\
(\%)\end{array}$ & Média & Moda & Mediana & Variância & Desvio & $\begin{array}{c}\text { Quadrado dos } \\
\text { desvios }\end{array}$ & $\begin{array}{c}\text { Desvio } \\
\text { Padrão }\end{array}$ \\
\hline $\mathbf{2 0 1 0}$ & $10,75 \%$ & 9,80 & Amodal & 10,38 & 8,27 & 0,95 & $(0,95)^{2}=0,90$ & 2,88 \\
\hline $\mathbf{2 0 1 1}$ & $11,00 \%$ & 9,80 & Amodal & 10,38 & 8,27 & 1,20 & $(1,20)^{2}=1,44$ & 2,88 \\
\hline $\mathbf{2 0 1 2}$ & $7,25 \%$ & 9,80 & Amodal & 10,38 & 8,27 & $(-2,55)$ & $(-2.55)^{2}=6,50$ & 2,88 \\
\hline $\mathbf{2 0 1 3}$ & $10,00 \%$ & 9,80 & Amodal & 10,38 & 8,27 & 0,20 & $(0,20)^{2}=0,04$ & 2,88 \\
\hline $\mathbf{2 0 1 4}$ & $11,70 \%$ & 9,80 & Amodal & 10,38 & 8,27 & 1,90 & $(1,90)^{2}=3,61$ & 2,88 \\
\hline $\mathbf{2 0 1 5}$ & $14,25 \%$ & 9,80 & Amodal & 10,38 & 8,27 & 4,45 & $(4,45)^{2}=19,81$ & 2,88 \\
\hline $\mathbf{2 0 1 6}$ & $13,75 \%$ & 9,80 & Amodal & 10,38 & 8,27 & 3,95 & $(3,95)^{2}=15,61$ & 2,88 \\
\hline $\mathbf{2 0 1 7}$ & $7,00 \%$ & 9,80 & Amodal & 10,38 & 8,27 & $(-2,80)$ & $(-2,80)^{2}=7,83$ & 2,88 \\
\hline $\mathbf{2 0 1 8}$ & $6,50 \%$ & 9,80 & Amodal & 10,38 & 8,27 & $(-3,30)$ & $(-3,30)^{2}=10,88$ & 2,88 \\
\hline $\mathbf{2 0 1 9}$ & $5,79 \%$ & 9,80 & Amodal & 10,38 & 8,27 & $(-4,01)$ & $(-4,01)^{2}=16,07$ & 2,88 \\
\hline
\end{tabular}

Fonte: Os dados dos valores foram extraídos da Figura 1.

Nota: Elaborada pelos autores.

Como se observa na tabela 1 , as taxas de juros SELIC nos últimos anos apresentaram muitas variações, sendo o maior aumento das taxas de juros se deu no ano de 2015, pois variou nos valores em (\%) de 11,7\% em 2014 para 14,25\% em 2015 , e a maior queda foi no ano de 2019 , variando de $13,75 \%$ em 2016 para $5,79 \%$ em 2019, obtendo nesse período um desvio padrão de 2,88.

$\mathrm{O}$ desvio padrão é demonstrado pela seguinte fórmula: média aritmética $(\mathrm{x}) \pm$ desvio padrão (dp). O cálculo do desvio padrão é realizado com base do valor positivo da raiz quadrada da variância. Portanto $D P=\sqrt{ }$ var.

Dessa forma, o desvio padrão aparece acompanhando a média aritmética, transmitindo o quanto verdadeiro esse valor é. Um baixo Desvio Padrão indica que os pontos dos dados (discrepância) tendem a estar próximos da média ou do valor 
esperado. O valor do desvio padrão, nesse cenário foi de 2,88 , e indica que os pontos dos dados entre 2015 e 2016 estariam dispersos e distantes da média por uma ampla gama de valores (RIBEIRO, 2019), então o desvio padrão referente à taxa de juros é alto.

Assim, os dados da medida central confirmam que quanto mais altos eram os juros no cenário dos anos 2015 e 2016, maior seria o Desvio Padrão, por isso, o governo teve que manter esforços para manter uma taxa de juros mais baixa e consequentemente teria futuramente um Desvio Padrão menor.

Chegou-se ao resultado do Desvio Padrão usando a fórmula: $D_{\rho}=\sqrt{ } 8,27=$ 2,88 observando alta discrepância entre os anos 2015 e 2016.

$\mathrm{Na}$ tabela 2, será apresentado a correlação dos valores das Medidas de tendência Central do IPCA, que é o índice oficial do Brasil para medir as metas da inflação. O IPCA é calculado mensalmente pelo IBGE e foi instituído com a finalidade de corrigir as expressões financeiras das empresas abertas.

Tabela 2: Medidas de Tendência Central dos Resultados das Taxas do IPCA no período de 2010 a 2019.

\begin{tabular}{c|c|c|c|c|c|c|c|c}
\hline $\mathbf{A n o}$ & $\begin{array}{c}\text { Valores } \\
(\%)\end{array}$ & Média & Moda & Mediana & Variância & Desvio & $\begin{array}{c}\text { Quadrado } \\
\text { dos desvios }\end{array}$ & $\begin{array}{c}\text { Desvio } \\
\text { Padrão }\end{array}$ \\
\hline $\mathbf{2 0 1 0}$ & $5,91 \%$ & 5,85 & 5,91 & 5,91 & 3,94 & 0,06 & $(0,06)^{2}=0,00$ & 1,98 \\
\hline $\mathbf{2 0 1 1}$ & $6,50 \%$ & 5,85 & 5,91 & 5,91 & 3,94 & 0,65 & $(0,65)^{2}=0,42$ & 1,98 \\
\hline $\mathbf{2 0 1 2}$ & $5,84 \%$ & 5,85 & 5,91 & 5,91 & 3,94 & $-0,01$ & $(-0,01)^{2}=0,00$ & 1,98 \\
\hline $\mathbf{2 0 1 3}$ & $5,91 \%$ & 5,85 & 5,91 & 5,91 & 3,94 & 0,06 & $(0,06)^{2}=0,00$ & 1,98 \\
\hline $\mathbf{2 0 1 4}$ & $6,41 \%$ & 5,85 & 5,91 & 5,91 & 3,94 & 0,56 & $(0,56)^{2}=0,31$ & 1,98 \\
\hline $\mathbf{2 0 1 5}$ & $10,67 \%$ & 5,85 & 5,91 & 5,91 & 3,94 & 4,82 & $(4,82)^{2}=23,19$ & 1,98 \\
\hline $\mathbf{2 0 1 6}$ & $6,29 \%$ & 5,85 & 5,91 & 5,91 & 3,94 & 0,44 & $(0,44)^{2}=0,19$ & 1,98 \\
\hline $\mathbf{2 0 1 7}$ & $2,95 \%$ & 5,85 & 5,91 & 5,91 & 3,94 & $-2,90$ & $(-2,90)^{2}=8,43$ & 1,98 \\
\hline $\mathbf{2 0 1 8}$ & $3,75 \%$ & 5,85 & 5,91 & 5,91 & 3,94 & $-2,10$ & $(-2,10)^{2}=4,43$ & 1,98 \\
\hline $\mathbf{2 0 1 9}$ & $4,31 \%$ & 5,85 & 5,91 & 5,91 & 3,94 & $-1,54$ & $(-1,54)^{2}=2,38$ & 1,98 \\
\hline
\end{tabular}

Fonte: Os dados dos valores foram extraídos da Figura 2.

Nota: Elaborada pelos autores.

De acordo com a tabela 2, referente ao IPCA registrado de 2010 a 2019 notase que a média dos valores é de $5,85 \%$. O desvio padrão obtido foi 1,98. Comparando 
o IPCA com o mesmo período 2015 e 2016 com as taxas de juros, o desvio padrão indicava que os valores de 2015 e 2016 ficaram muito acima do esperado, enquanto o IPCA de 2015 foi de 10,67\%, o ano de 2016 terminou com 6,29\%, isso se deu a consequência das altas taxas de juros apresentadas na Figura1 e na Tabela1, o que inibiu o consumo das famílias.

Comparando os anos de 2016 e 2017, observou-se uma queda nos percentuais do IPCA, o mesmo também se obteve na taxa de juros referente a esses anos, enquanto 2016 o IPCA foi de 6,29\%, em 2017 o IPCA caiu para 2,95\% tendo as mesmas observações quando comparadas nas taxas de juros apresentadas no Figura 1 e Tabela1. Nota-se que nos anos de 2017, 2018 e 2019 começaram as altas do IPCA e a baixa dos preços ao consumidor, terminando 2017 com 2,95\%, 2018 com $3,75 \%$ e fechando 2019 com 4,31\%.

Pode-se comprovar pelo desvio padrão que está mais baixo no IPCA em relação à taxa de juros, o que demonstra que o poder de compra a partir de 2017 começou a ser estimulado pela economia.

Assim, o Brasil teve uma inflação elevada em detrimento aos anos anteriores, 2013 a 2016.

Nesse cenário, foi encontrado um Desvio Padrão de 1,98 que é o reflexo dos preços aos consumidores no país no período analisado e seu cálculo se deu por meio da fórmula Desvio Padrão: $D_{\rho}=\sqrt{3}, 94=1,98$. Os resultados apontam para uma média discrepância entre 2017 e 2019, sugerindo uma retomada econômica com poder de compra pelos brasileiros.

A Tabela 3 revela se refere à taxa de desemprego no Brasil. Determina a taxa de desemprego pelo IBGE e os valores apresentados na tabela 3 foram determinados a partir das pesquisas realizadas com a população economicamente ativa no País.

Tabela 3: Medidas de Tendência Central dos Resultados das Taxas do Desemprego no período de $\mathbf{2 0 1 0}$ a 2019

\begin{tabular}{c|c|c|c|c|c|c|c|c}
\hline Ano & $\begin{array}{c}\text { Valores } \\
(\%)\end{array}$ & Média & Moda & Mediana & Variância & Desvio & $\begin{array}{c}\text { Quadrado dos } \\
\text { desvios }\end{array}$ & $\begin{array}{c}\text { Desvio } \\
\text { Padrão }\end{array}$ \\
\hline $\mathbf{2 0 1 0}$ & 6,7 & 8,28 & Amodal & 6,8 & 8,98 & $(-1,58)$ & $(-1,58)^{2}=2,50$ & 3,00 \\
\hline $\mathbf{2 0 1 1}$ & 6,0 & 8,28 & Amodal & 6,8 & 8,98 & $(-2,28)$ & $(-2,28)^{2}=5,20$ & 3,00 \\
\hline $\mathbf{2 0 1 2}$ & 5,5 & 8,28 & Amodal & 6,8 & 8,98 & $(-2,78)$ & $(-2,78)^{2}=7,73$ & 3,00 \\
\hline
\end{tabular}




\begin{tabular}{c|c|c|c|c|c|c|c|c}
\hline $\mathbf{2 0 1 3}$ & 5,4 & 8,28 & Amodal & 6,8 & 8,98 & $(-2,88)$ & $(-2,88)^{2}=8,29$ & 3,00 \\
\hline $\mathbf{2 0 1 4}$ & 4,3 & 8,28 & Amodal & 6,8 & 8,98 & $(-3,48)$ & $(-3,48)^{2}=12,11$ & 3,00 \\
\hline $\mathbf{2 0 1 5}$ & 6,9 & 8,28 & Amodal & 6,8 & 8,98 & $(-1,38)$ & $(-1,38)^{2}=1,90$ & 3,00 \\
\hline $\mathbf{2 0 1 6}$ & 11,5 & 8,28 & Amodal & 6,8 & 8,98 & 3,22 & $(3,22)^{2}=10,37$ & 3,00 \\
\hline $\mathbf{2 0 1 7}$ & 11,8 & 8,28 & Amodal & 6,8 & 8,98 & 3,52 & $(3,52)^{2}=12,39$ & 3,00 \\
\hline $\mathbf{2 0 1 8}$ & 12,3 & 8,28 & Amodal & 6,8 & 8,98 & 4,02 & $(4,02)^{2}=16,16$ & 3,00 \\
\hline $\mathbf{2 0 1 9}$ & 11,9 & 8,28 & Amodal & 6,8 & 8,98 & 3,62 & $(3,62)^{2}=13,10$ & 3,00 \\
\hline
\end{tabular}

Fonte: Os dados dos valores foram extraídos da Figura 3.

Nota: Elaborada pelos autores.

De acordo com as análises dos dados estatísticos e históricos das taxas de desemprego, pode-se perceber que o desvio padrão representa um índice de 3,0 acompanhando a média de $8,28 \%$, levando em consideração que essa diferença eleva gradativamente o resultado, porém, não sendo um fator positivo, pois quanto mais próximo a taxa estiver de zero, menor será o grau de desemprego ao longo dos anos, porém, observa-se o contrário, ou seja, um desvio padrão alto, elevando assim as altas taxas de desemprego que o país atravessou.

O desvio padrão foi calculado ano a ano pela seguinte fórmula: Desvio Padrão $=D_{\rho}=\sqrt{ } 8,89=3,00$ e percebeu-se que a partir do ano 2010 até 2015, as taxas de desempregos mantiveram-se em um patamar quase estável, com poucas variações. Já a partir de 2016 até 2019, percebe-se claramente que a taxa de desemprego cresceu vertiginosamente, tendo um desvio padrão no período de 3,00, o que indica que o país esteve atravessando um período de altas taxas de desemprego, ou seja, um desvio padrão baixo indicaria que os pontos dos dados (dispersão) tenderiam a estar próximos da média ou do valor esperado.

Não obstante, um desvio padrão elevado como apresenta-se na tabela 3 indica que os pontos dos dados estariam dispersos por uma ampla gama de variáveis e isso corrobora que nos anos 2016 a 2019 com as altas taxas de desemprego, consequentemente afetou drasticamente o consumo e a renda no país.

\section{Conclusão}


A proposta deste trabalho teve como objetivo principal analisar o cenário macroeconômico do Brasil no período compreendido entre os anos de 2010 até 2019, bem como apresentar as perspectivas econômicas que geraram a crise financeira.

Em consideração aos fatos observados afirma-se que o objetivo foi alcançado, pois notou-se a fragilidade de um sistema de governo instável (no Brasil) e refletiu diretamente na economia e consequentemente na inflação que por seu lado foi a causadora dos principais problemas abordados neste trabalho.

Da mesma forma os demais indicadores estudados, IPCA e a Taxa de Desemprego, ambos se oriundam das Taxas de Juros e assim como foram demonstrados nas figuras 1, 2 e 3 é possível apontar que com o início de uma crise econômica desde 2013 até 2016 as Taxas de Juros aumentaram com a finalidade de manter a inflação controlada e evitar que fossem feitos empréstimos durante o período; porém a partir de 2017 com uma economia mais otimista e com expectativas de mudança, houve queda nas Taxas de Juros até 2019, com exceção de 2018 que voltaram a cair; e mediante aos resultados houve incentivo aos investimentos tendo em vista a queda da inflação.

No cenário de 2015 e 2016, o desvio padrão dos Juros indicaram que os dados tenderam a estarem mais distantes do valor estimado, ou seja, mais altos. Para uma menor taxa de juros entre as variáveis macroeconômicas, destacam-se a recuperação da economia, o controle da inflação, a estabilidade da dívida pública, a capacidade do governo de pagar os juros da dívida com recursos fiscais, a sustentabilidade do balanço de pagamentos e a normalidade política.

Como resultado da pesquisa, ficou explícito por meio das análises da figura 2 e dos cálculos da tabela 2 que ao analisar o IPCA o país passou por crise econômica de 2013 a 2016; e que a partir de 2017 até 2019 demonstram consideráveis recuperação econômica.

Porém, pode-se inferir que durante a menção das Taxas de Desempregos apresentados na figura 3, realizou-se o cruzamento dos dados na tabela 3 e apontaram resultados diferentes decorrentes das consequências a longo prazo, gerando uma crise econômica comprovadas pelas análises da tendência central.

Ao considerarmos as figuras e as tabelas 1, 2 e 3, é nítido observar que houve uma discrepância dos indicadores analisados entre os anos de 2010 a 2015 em relação aos anos de 2016 a 2018 e isso ocorreram devido às iniciativas em sua grande 
maioria das empresas privadas, por não conseguirem se sustentar mediante a crise, sendo inviável manter os funcionários diante da baixa taxa de produção/venda de bens e serviços o que resultaram em demissões em massa.

Nessa linha, o que se observou ainda foi uma queda de todos os indicadores analisados nos anos de 2018 e 2019 quando a economia voltou a se estabilizar e com isso houve a diminuição da Taxa de Desemprego que veio crescendo progressivamente desde 2016.

Entre as variáveis macroeconômicas analisadas, podem ser citadas as contribuições como intervenções do governo no mercado de crédito, a segurança jurídica, a confiança nas instituições. Todos esses fatores, isoladamente ou em conjunto, influenciam nas taxas dos juros.

Por fim, concluiu-se que a economia Brasileira passou por anos de crises e instabilidades, o que resultou em Inflação alta, desvalorização do Real e taxas de desemprego elevadas.

Ainda de acordo com os resultados analisados, percebeu-se que há uma tendência da recuperação da economia para os anos subsequentes em todos os aspectos, como o que sugere como exemplo a reforma da previdência sancionada pelo atual Presidente em novembro de 2019 e a reforma administrativa ainda em curso no Congresso Nacional e outras.

Faz-se observar que o ano de 2020 não entrou nas estimativas dos parâmetros fiscais, pois o ano não se encerrou e o cenário estimado nesse trabalho foi anual.

Esta pesquisa não teve a intenção de esgotar seus estudos, suas análises e suas interpretações, porém sugere-se que novas pesquisas sejam realizadas nos anos vindouros para confirmarem o que esta revelou.

Por fim, esse estudo não se esgota por si só e, porém, teve um desdobramentos e motivações de outros estudos já publicados pela Revista Multidisciplinar do Nordeste Mineiro de autoria do mesmo autor deste estudo, os quais citam-se:

i) Dificuldades da Inclusão de Pessoas com Deficiência no Mercado de Trabalho;

ii) Liderança: seu papel visando o clima e a cultura nas organizações; 
iii) Desmotivação no ambiente de trabalho: fatores que geram medidas para a reversão;

iv) Inclusão de pessoas com deficiência no mercado de trabalho: a relevância das atividades mercadológicas e sociais;

v) O planejamento estratégico e o seu papel para a sobrevivência das micro e pequenas empresas;

vi) A cultura organizacional e sua relação com o desempenho das empresas e;

vii) Um estudo sobre o controle do estoque hospitalar com ênfase no gerenciamento sobre os custos e a armazenagem.

\section{Referências}

AGÊNCIA BRASIL. Em dezembro, IPCA foi de 1,15\% e acumulou alta de 4,31\% em 2019. Disponível em < https://agenciadenoticias.ibge.gov.br/agencia-sala-deimprensa/2013-agencia-de-noticias/releases/26619-em-dezembro-ipca-foi-de-1-15e-acumulou-alta-de-4-31-em2019\#: :text=O\%20IPCA\%20fechou\%202019\%20com,3\%2C75\%25\%20de\%20201 8.\&text=O\%20resultado\%20de\%202019\%20foi,p.p.\%20no\%20acumulado\%20do\%2 0ano. > Acesso em 07/09/2020.

Taxa de desemprego cai no país e fecha 2019 em 11,9\%. Disponível em < https://agenciabrasil.ebc.com.br/economia/noticia/2020-01/taxa-de-desempregono-pais-fecha-2019-em-119 > Acesso em 30/08/2019.

\section{BALASSIANO, Marcel. Retrospecto de 2018 e Perspectivas para 2019 na} Economia. Instituto Liberal, 2018. Disponível em: $<$ https://www.institutoliberal.org.br/blog/retrospecto-de-2018-e-perspectivas-para2019-na-economia/>. Acesso em: 30/08/2019.

BARBOSA FILHO, F. Nota sobre evolução da produtividade no Brasil. Nota técnica. IBRE-FGV, 2014. 
CAMPBELL, D.T., \& STANLEY, J.C. Delineamentos experimentais e quase experimentais de pesquisa. São Paulo: E.P.U.1979.

CARVALHO, Leonardo Mello de. Atividade econômica: desempenho do PIB. IPEA, 2018. Disponível em: <http://www.ipea.gov.br/cartadeconjuntura/wpcontent/uploads/2018/08/CC40_Atividade_PIB3.pdf>. Acesso em: 30/08/2019.

CEMEC. Endividamento das empresas brasileiras: metade das empresas não gera caixa para cobrir despesas financeiras em 2015/2016. Nota CEMEC 06/2016, agosto de 2016.

CONTANDRIOPOULOS, A. P., CHAMPAGNE, F., POTVIN, L., DENIS, J. O., \& BOYLE, P. Saber preparar uma pesquisa. São Paulo: Hucitec Abrasco. 1994.

FOCUS - Relatório de Mercado - Banco Central do Brasil - Brasil. 2019.

GIAMBIAGI, F.; ALMEIDA JR., M. A retomada do crescimento. Rio de Janeiro: Elsevier, 2017.

IBGE - Instituto Brasileiro de Geografia e Estatística. Indicadores IBGE : contas nacionais trimestrais. 2015. Disponível em:

$<$ https://www.ibge.gov.br/estatisticas/economicas/contas-nacionais/9300-contasnacionais-trimestrais.html?edicao=20920\&t=publicacoes $>$. Acesso em: 30/08/2019.

. Indicadores IBGE: contas nacionais trimestrais. 2017. Disponível em: <https://biblioteca.ibge.gov.br/visualizacao/periodicos/2121/cnt_2017_1tri.pdf>. Acesso em 30/08/2019.

Índice nacional de preços ao consumidor amplo - IPCA. Disponível em <https://www.ibge.gov.br/estatisticas/economicas/precos-e-custos/9256-indicenacional-de-precos-ao-consumidor-amplo.html?=\&t=0-que-e>. Acesso em 29/08/2019. 
Sistema nacional de índices de preços ao consumidor: métodos de cálculo. 7. ed. 2013.Disponível em <https://biblioteca.ibge.gov.br/visualizacao/livros/liv65477.pdf>. Acesso em 29/08/2019.

KANCZUK, Fábio. Juros reais e ciclos reais brasileiros. SciELO. FEA/USP, 2002. Disponível em: <http://www.scielo.br/scielo.php?pid=S0034-

71402002000200003\&script=sci_arttext\&tIng=pt> Aceso em: 30/08/2019

MARTINS, Gabriel Gondim et al. Conjuntura Econômica: Pós Eleições 2018. INSPER, Jr. Consulting. 2018. Disponível em:

<http://www.insperjr.com.br/uploads/arquivos/2018/12/panorama-de-mercadoconjuntura-economica-1544557608.pdf>. Acesso em: 30/08/2019.

PAULA, Luiz Fernando de; PIRES, Manoel. Crise e Perspectivas Para a Economia Brasileira. SciELO, 2017. Disponível em:

$<$ http://www.scielo.br/scielo.php?script=sci_arttext\&pid=S010340142017000100125>. Acesso em: 29/08/2019.

RIBEIRO, A. G. Medidas de dispersão: variância e desvio padrão; Brasil Escola. Disponível em: https://brasilescola.uol.com.br/matematica/medidas-dispersaovariancia-desvio-padrao.htm. Acesso em agosto de 2019.

SELLTIZ, C., WRIGHTSMAN, L. S., \& COOK, S. W. Métodos de Pesquisa nas Relações Sociais. (Vol. 1). São Paulo: EPU.1976.

SERRANO, Franklin. Juros, câmbio e o sistema de metas de inflação no Princípios, 2010. Disponível em: <http://www.scielo.br/scielo.php?pid=S010131572010000100004\&script=sci_arttext\&tIng=pt>. Aceso em: 30/08/2019

SILVEIRA, Daniel; ALVARENGA, Darlan. Setor de serviços avança $1,2 \%$ e tem melhor agosto desde 2011, aponta IBGE, G1. Rio de Janeiro e São Paulo, 
16/10/2018. Disponível em:

<https://g1.globo.com/economia/noticia/2018/10/16/setor-de-servicos-cresce-12-emagosto-aponta-ibge.ghtml>. Acesso em: 30/08/2019

TINOCO, Guilherme; GIAMBIAGI, Fábio. O Crescimento da Economia Brasileira. BNDES, 2018. Disponível em:

$<$ https://web.bndes.gov.br/bib/jspui/bitstream/1408/14760/1/Perspectivas\%2020182023_P.pdf>. Acesso em: 29/08/2019.

TREVIZAN, Karina. Projeções para o PIB do ano pioram após greve dos caminhoneiros, G1. 18/07/2018. Disponível em:

<https://g1.globo.com/economia/noticia/2018/07/18/projecoes-para-o-pib-do-anopioram-apos-greve-dos-caminhoneiros.ghtml>. Acesso em: 30/08/2019. 